\title{
Memory psychophysics for taste
}

\author{
DANIEL ALGOM and LAWRENCE E. MARKS \\ John B. Pierce Foundation Laboratory and Yale University \\ New Haven, Connecticut
}

\begin{abstract}
Subjects made quantitative judgments of taste stimuli (various concentrations of sucrose), which were either presented physically for a perceptual estimation or represented symbolically for a memory estimation. The perceived and remembered intensities were found to be related to the referent sucrose concentrations by power functions with similar exponents. This constancy of exponents contrasts with the lower memory exponents reported for other sensory continua, as well as with predictions based on theories of memory-based magnitude judgments. These results may further suggest a unique role for memory in chemosensation.
\end{abstract}

Psychophysics is the science that explicates the functional relations between physical stimuli and the resulting sensory responses. Memory psychophysics, or mnemophysics, is the branch of psychophysics that deals with quantitative relations between physical stimuli and their remembered sensory properties. Although mnemophysics has been applied successfully to visual continua such as remembered area, remembered volume, and remembered linear extent, virtually nothing is known about the rules that govern memory for intensity in the chemical senses.

Investigations over the past two decades attest to the unique properties of chemosensory memory. With flavors, conditioned aversion may be complete after a single trial, yet it lasts virtually forever (Logue, 1985). With odors, recognition endures, outperforming recollection in other sensory departments (see Cain, 1988; Engen, 1987). However, research to date has dealt almost exclusively with qualitative aspects of memory for odors, and virtually no studies have been performed on memory for taste (but see Moyer, Sklarew, \& Whiting, 1982, for some preliminary results on sweetness).

The present experiment is an early foray into the realm of memory of taste intensity. In perception, the psychophysical relation for taste generally conforms to a power function with exponents both below and above unity (Bartoshuk, 1988). Here, we ask how remembered taste magnitudes depend on referent physical intensities (concentrations). Do memory scale values map onto their physical referents by means of the same functional relation (power transform) as do perceptual scale values? And if so, do the same parameters (exponents) govern perceptual and memory functions? Standard psychophysical methods and analyses can be used with both perceptual and memory types of judgments, the only procedural difference being that taste stimuli are in the one case physically presented, in the other, symbolically represented.

The research reported in this paper was supported by NIH Grant NS16993. The authors thank Scott Masters for his assistance. Correspondence may be addressed to Daniel Algom at the John B. Pierce Foundation Laboratory, 290 Congress Avenue, New Haven, CT 06519.

\section{METHOD}

\section{Subjects}

Thirty young men and women, paid volunteers from the Yale community, served in the experiment.

\section{Stimuli and Procedure}

The experiment comprised two sessions. In the first session, the subjects learned to associate colors (displayed on the RGB monitor of an AMIGA microcomputer) with each of five different concentrations of sucrose $(.028, .083, .19, .42$, and $.69 \mathrm{M})$ dissolved in deionized water. The five colors-blue, green, brown, yellow, and red (matched for saturation)-were randomly assigned to the sucrose concentrations across subjects. The stimuli were presented at room temperature $\left(\sim 22^{\circ} \mathrm{C}\right)$, in cups containing approximately $10 \mathrm{ml}$. Before tasting each stimulus, the subjects rinsed their mouths thoroughly with distilled water. Intertrial intervals were at least $1 \mathrm{~min}$, in order to minimize adaptation.

For the first two presentations of the stimulus series (first concentration series in ascending order, second in descending order), the experimenter both showed the color (on the monitor) and spelled out its name (e.g., RED) as each taste solution was presented. In the subsequent trials, the subject had to supply the color name as each solution was tasted. After the subject's response, the correct color and its name were displayed, regardless of the response. The order of presentation was random and different for each subject. The trials continued until the following criterion was reached: either (1) three series out of five were completed with no errors, or (2) four consecutive series were completed with no more than one error in each series. These learning sessions took about $40-50$ min per subject. Five subjects failed to reach the criterion, but all fell short by no more than one erroneous identification.

After an interval of $24 \mathrm{~h}$, the subjects returned for a judgmental session. Each subject was randomly assigned to either a perceptual or a memory condition. In the perceptual condition, actual sucrose solutions were presented one at a time for judgment. In the memory condition, the subjects also made judgments of taste intensity, but no actual taste solutions were presented; instead, the intensities were represented by their previously learned colors. As the colors were shown and named in a random order, the subjects tried to imagine each stimulus concentration and then estimate its intensity. At the end of the testing, the subjects in both groups tasted a new stimulus, selected from the middle of the original range $(.28 \mathrm{M})$, and estimated its intensity.

The method used throughout was free-modulus magnitude estimation. The subjects were instructed to assign to the first stimulus whatever number seemed most appropriate to represent its intensity, then to assign proportional numbers to succeeding stimuli, using whole numbers, decimals, and fractions as needed.

\section{RESULTS AND DISCUSSION}

The magnitude estimates of taste given to each stimulus were averaged geometrically; these means are plot- 


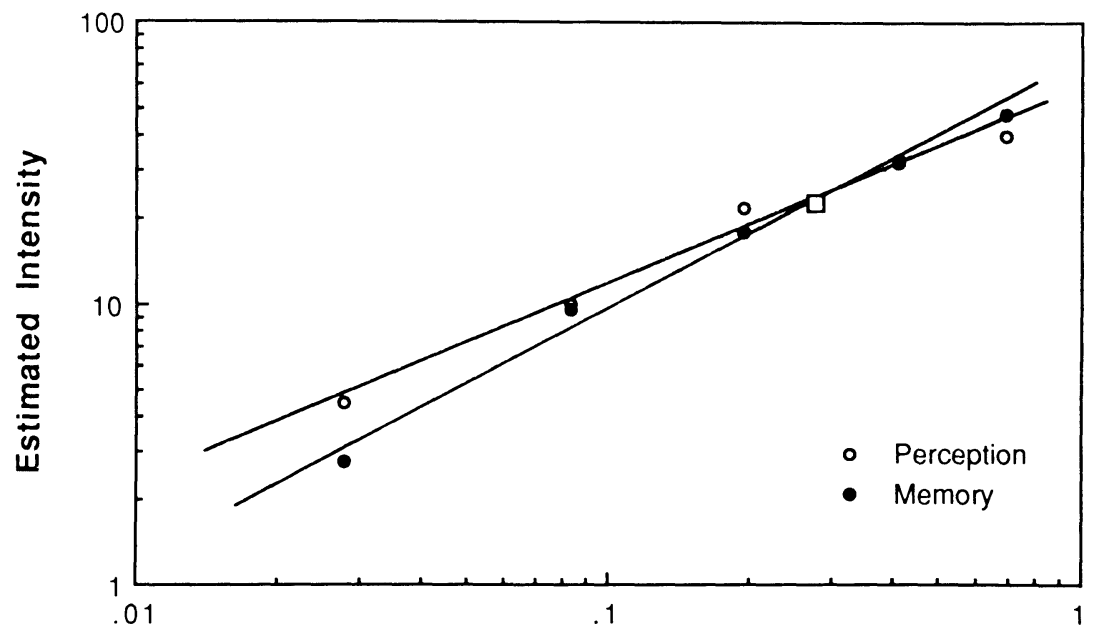

Molar Concentration Sucrose

Figure 1. Perceptual and memory psychophysical functions for taste intensity. The square stands for the common $(.28 \mathrm{M})$ perceptually judged stimulus that yielded virtually identical means in the two groups.

ted in Figure 1, as a function of concentration level. A separate function is drawn for each judgmental condition. The most striking feature of each set of points is the excellent fit by a power relation (both sets approximate straight lines in the double logarithmic coordinates). The Pearson $r^{2}$ values equal 0.984 and 0.992 , for the perceptual and memory conditions, respectively. The slopes (exponents of the power functions) are 0.70 for perception and 0.87 for memory. If the individual subjects' exponents are pooled, the means are .66 and .83 , respectively; this difference is not significant $[t(28)=2.013$, $p>0.05]$. To the extent that the slopes do differ, the observed relation deviates even more from previous findings that typically report a smaller memory exponent.

The reappearance of the power relation for remembered intensities is just as important as the exact values of the exponents or their relation. This study provides the first demonstration of a lawful connection between remembered taste intensity and referent physical concentration. Moreoever, the data suggest that the psychophysical and mnemophysical power functions are governed by roughly the same exponent. This latter finding contrasts sharply with mnemophysical results from other modalities. For visual area, for example, memory exponents are significantly smaller than their perceptual counterparts (see, e.g., Algom, Wolf, \& Bergman, 1985; Kerst \& Howard, 1978; Moyer, Bradley, Sorensen, Whiting, \& Mansfield, 1978; Wolf \& Algom, 1987). This is typical. Memory exponents have been smaller than corresponding perceptual exponents when the perceptual exponent is smaller than unity. Therefore, the uniformity of exponents in taste perception and memory may pinpoint a distinctively chemosensory property limited only to remembered taste and, possibly, odor.

Table 1 gives the individual results. The subjects in both groups were consistent in conforming to a power rela- tion, demonstrating no appreciable differences across mode of response generation. Note that even the variability of exponents is comparable in the two groups $(\sigma=.224$ and .227 , respectively, for perception and memory).

A final important feature of the data deserves mention. Despite the fact that different groups of subjects participated in the perceptual and the memory conditions, no significant differences in the absolute magnitude of judgments were found at any of the experimental values tested (including the common additional stimulus whose judged magnitude was virtually the same as the rest).

Two rival theoretical formulations have been suggested to account for properties of memory-based magnitude judgments (e.g., Algom et al., 1985). Neither explana-

Table 1

Parameters of Perceptual and Memory Psychophysical Functions, Individually for Thirty Subjects

\begin{tabular}{|c|c|c|c|c|c|}
\hline \multirow[b]{2}{*}{$S$} & \multicolumn{2}{|c|}{ Perception } & \multirow[b]{2}{*}{$S$} & \multicolumn{2}{|c|}{ Memory } \\
\hline & $b$ & $r^{2}$ & & $b$ & $r^{2}$ \\
\hline 1 & 0.603 & 0.771 & 16 & 0.940 & 0.860 \\
\hline 2 & 0.412 & 0.946 & $17^{*}$ & 0.896 & 0.966 \\
\hline 3 & 0.490 & 0.981 & $18^{*}$ & 1.090 & 0.975 \\
\hline 4 & 0.391 & 0.575 & 19 & 0.704 & 0.986 \\
\hline 5 & 0.929 & 0.873 & 20 & 1.204 & 0.848 \\
\hline 6 & 0.634 & 0.576 & 21 & 0.627 & 0.974 \\
\hline 7 & 0.662 & 0.959 & 22 & 0.874 & 0.933 \\
\hline 8 & 0.414 & 0.702 & $23 *$ & 0.655 & 0.957 \\
\hline 9 & 0.799 & 0.932 & 24 & 0.488 & 0.975 \\
\hline 10 & 0.762 & 0.976 & 25 & 0.473 & 0.960 \\
\hline 11 & 0.995 & 0.973 & 26 & 1.061 & 0.974 \\
\hline $12 *$ & 0.493 & 0.848 & 27 & 0.822 & 0.940 \\
\hline 13 & 0.822 & 0.971 & 28 & 1.112 & 0.939 \\
\hline 14 & 0.427 & 0.943 & 29 & 0.852 & 0.961 \\
\hline $15^{*}$ & 1.049 & 0.930 & 30 & 0.652 & 0.977 \\
\hline
\end{tabular}

Note $-\mathrm{S}=$ subject, $b=$ power function's exponent. $*$ Did not pass learning criterion. 
tion, however, can accommodate our results. According to the reperception hypothesis, perception and memory perform identical (power) transformations on the input data. Because the input to memory processing has already been transformed perceptually (by exponent $b$ ), the memory exponent should reflect two transformations, and thus equal $b^{2}$. The alternative uncertainty hypothesis posits that greater uncertainty causes subjects to constrict the range of responses, thereby producing an attenuated memory exponent. Note that both theories predict a more compressive memory function for perceptual dimensions that are characterized by a smaller-than-unity power function exponent like the one found here. Clearly, the present findings are incompatible with either formulation.

A modified version of the reperception hypothesis can, however, account for the present results as well as for other similar data (e.g., Kemp, 1988; Moyer et al., 1982; Osaka, 1987). We propose a two-stage model of judgment (cf. Algom \& Marks, 1984), according to which the sizes of the measured exponents $\boldsymbol{P}$ (for perception) and $M$ (for memory) vary because of response transformations imposed by nonlinear judgmental processes (transformations by a power function with exponent $c$ ). Mathematically, $R_{\mathrm{p}}=k S^{b c}$ for perception, and $R_{\mathrm{m}}=k S^{b b c}$ for memory. The ratio of the measured exponents $M / P$ (i.e., $b b c / b c$ ) yields the underlying unbiased perceptual exponent $b$. This exponent, which in the present case equals $1.26(.87 / .70)$, should be distinguished from the observed exponent $P(b c=.70)$ which is confounded by nonlinear biases. Interestingly enough, exponents reported for sweetness of sucrose often are in the vicinity of 1.3 (see, e.g., Stevens, 1969).

The two-stage model implies that the near equivalence of perceptual and memory exponents found here is perhaps fortuitous. Alternatively, we may interpret the present results to mean simply what they say: Memory for taste intensity is lawfully related to the referent concentrations; and, at least for a day, memory judgments are practically indistinguishable from immediate judgments, when ap- plied to the same taste stimuli. The results reinforce the common impression that memory plays a unique role in chemosensation in yet another respect.

\section{REFERENCES}

Algom, D., \& Marks, L. E. (1984). Individual differences in loudness processing and loudness scales. Journal of Experimental Psychology: General, 113, 471-493.

Algom, D., Wolf, Y., \& Bergman, B. (1985). Integration of stimulus dimensions in perception and memory: Composition rules and psychophysical relations. Journal of Experimental Psychology: General, 114, 451-471.

Bartoshuk, L. M. (1988). Taste. In R. C. Atkinson, R. J. Herrnstein G. Lindzey, \& R. D. Luce (Eds.), Stevens' handbook of experimental psychology: Vol. 1. Perception and motivation (pp. 461-499). New York: Wiley.

CaIN, W. S. (1988). Olfaction. In R. C. Atkinson, R. J. Herrnstein G. Lindzey, \& R. D. Luce (Eds.), Stevens' handbook of experimental psychology: Vol. 1. Perception and motivation (pp. 409-459). New York: Wiley.

ENGEN, T. (1987). Remembering odors and their names. American Scientist, 75, 497-503.

KEMP, S. (1988). Memorial psychophysics for visual area: The effect of retention interval. Memory \& Cognition, 16, 431-436.

Kerst, S. M., \& Howard, J. H., JR. (1978). Memory psychophysics for visual area and length. Memory \& Cognition, 6, 327-335.

Logue, A. W. (1985). Conditioned food aversion in humans. Annals of the New York Academy of Sciences, 443, 316-329.

Moyer, R. S., Bradley, D. R., Sorensen, M. H., Whiting, J. D., \& MANSFIELD, D. P. (1978). Psychophysical functions for perceived and remembered size. Science, 200, 330-332.

Moyer, R. S., Sklarew, P., \& Whiting, J. (1982). Memory psychophysics. In H. G. Geissler \& P. Petzold (Eds.), Psychophysical judgment and the process of perception (pp. 35-46). Berlin: VEB Deutscher Verlag der Wissenschaften.

OsaKA, N. (1987). Memory psychophysics for pyridine smell scale. Bulletin of the Psychonomic Society, 25, 56-57.

Stevens, S. S. (1969). Sensory scales of taste intensity. Perception \& Psychophysics, 6, 302-308.

Wolf, Y., \& Algom, D. (1987). Perceptual and memorial constructs in children's judgments of quantity: A law of across-representational invariance. Journal of Experimental Psychology: General, 116, 381-397.

(Manuscript received October 3, 1988.) 